\title{
Fatal Case of Bacteremia Caused by an Atypical Strain of Corynebacterium mucifaciens
}

\author{
Vlademir Vicente Cantarelli, ${ }^{1,2,3}$, \\ Teresa Cristina. Z. Brodt ${ }^{2}$, \\ Carina Secchi², Everton Inamine ${ }^{2}$, \\ Fabiana de Souza Pereira ${ }^{1}$ and Diogo Andre Pilger ${ }^{1}$ \\ Molecular Biology $y^{1}$ and Microbiology $y^{2}$ sections, Weinmann Laboratory \\ LTDA, Porto Alegre; Feevale Universitary Centre ${ }^{3}$, Novo Hamburgo, \\ RS, Brazil
}

\begin{abstract}
Corynebacterium species have often been considered normal skin flora or contaminants; however, in recent years they have been increasingly implicated in serious infections. Moreover, many new species have been discovered and old species renamed, especially after molecular biology techniques were introduced. Corynebacterium mucifaciens is mainly isolated from blood and from other normallysterile body fluids; it forms slightly yellow, mucoid colonies on blood agar. We report a fatal case of bacteremia due to an atypical strain of $C$. mucifaciens. This strain had atypical colony morphology; analysis of the 16S rRNA gene was used to define the species.
\end{abstract}

Key Words: Bacteremia, blood infection, Corynebacterium, Corynebacterium mucifaciens.

Determining the clinical significance of corynebacteria isolated from clinical specimens is often challenging for clinical microbiologists. For many years, these organisms were disregarded as skin contaminants and no further attempt to identify them at the species level was made. However, they have increasingly been recognized as important human pathogens, often acting as opportunistic pathogens in immunocompromised or severely-ill patients. Identification to the species level is recommended if more than one blood culture bottle tests positive, the patient has symptoms compatible with bacteremia, and no other pathogenic organisms are present [1]. Corynebacterium mucifaciens is a newly-described species that typically grows on blood agar plates, forming slightly yellow and mucoid colonies [2]. Identification can be made with standard biochemical tests or with commercial identification systems, such as API Coryne. However, differentiation from closely-related species is better accomplished with molecular biology techniques, such as sequencing of the $16 \mathrm{~S}$ rRNA gene.

Antimicrobial susceptibility tests for Corynebacterium species lack standardization, and empirical therapy is often used to treat patients known to be infected by Corynebacterium spp. With few exceptions, such as $C$. jeikeium, all Corynebacterium spp. show good clinical response to penicillin or vancomycin [3]. In the case of $C$. mucifaciens, beta-lactam antibiotics and aminoglycosides appear to have good activity [1].

We report isolation of an atypical strain of C. mucifaciens recovered from multiple blood cultures made from an elderly female patient.

Received on 11 October 2006; revised 21 November 2006.

Address for correspondence: Dr.. Vlademir Vicente Cantarelli. Weinmann Laboratório LTDA. Ramiro Barcelos-910/4 andar, Zip code: 90035-001 Porto Alegre, RS, Brazil. Phone: +55-51-33143850 Fax: +55-51-3311-7813. E-mail: vcantarelli@weinmann.com.br

The Brazilian Journal of Infectious Diseases 2006;10(6):416-418. (C) 2006 by The Brazilian Journal of Infectious Diseases and Contexto Publishing. All rights reserved.

\section{Case Report}

An 81-year-old woman was admitted to the hospital after a bad fall. Her past clinical history included: diabetes, ischemic cardiopathy, pulmonary fibrosis and an esophagic tumor that had been treated with radiotherapy a couple of years before. Laboratory findings revealed a white-blood-cell count of $15,500 / \mathrm{mm}^{3}$, with $85 \%$ neutrophils ( $7 \%$ bands); the platelet count was $277,000 / \mathrm{mm}^{3}$, with no marked alteration in the erythrocytic series. Blood glucose was $213 \mathrm{mg} / \mathrm{dL}$. Three blood samples from three separate venipunctures were submitted to the clinical microbiology laboratory for aerobic culture and were continuously monitored for any bacterial growth, using the BactAlert instrument (Organon-Teknika). All samples became positive after three days of incubation, and a Gram stain revealed Gram-positive, non-spore-forming diphtheroid rods. The patient died before complete identification of the organism was available.

The organism was subcultured on both chocolate and plain sheep blood agar (bioMérieux), revealing approximately 1-mm diameter whitish non-mucoid colonies after $24 \mathrm{~h}$ incubation. Colonies were about the same size after $72 \mathrm{~h}$ incubation (Figure 1). Catalase reaction tested positive. The first attempt to identify the organism was made using APICoryne (BioMeriéux) strips, following the manufacturer's instructions. The numerical code (2100104) suggested $C$. mucifaciens $[1,4]$.

In disagreement with published information on $C$. mucifaciens, our strain produced smooth-white colonies (Figure 1), instead of yellow and mucoid colonies [2]. This prompted us to check our initial identification with $16 \mathrm{~S}$ rRNA gene sequence analysis. DNA was obtained using a modified guanidine-isothiocyanate method [5], and the 16S rRNA gene (ca. $1500 \mathrm{bp}$ ) was amplified using primers 285 (5' GAGAGTTTGATCCTGGCTCAG - 3') and 261 (5' AAGGAGGTGATCCAGCCGCA - 3') [6] and a GeneAmp ${ }^{\text {TM }}$ PCR reagent kit (Applied Biosystems). PCR product was cleaned using shrimp alkaline phosphatase and Exonuclease I (USB) and sequenced using primer 806-F (5' - 
Figure 1. A. Small white colonies of Corynebacterium mucifaciens on sheep blood agar after $72 \mathrm{~h}$ of incubation. 1 . B. higher magnification of colonies from the same plate.

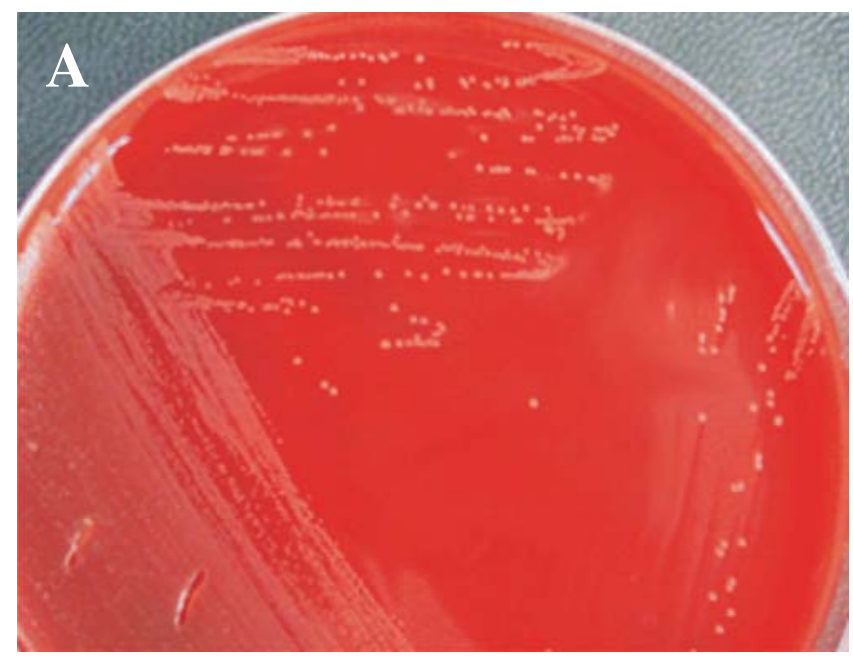

ATtAGATACCCTGGTAGTCC - 3') and the Big Dye terminator DNA Sequencing kit (Applied Biosystems), as described elsewhere [7]. A partial sequence (562 bp, corresponding to nucleotides 765 to 1326 ) was aligned and compared with other available 16S rRNA gene sequences using the bioinformatics bacterial identification tool (BIBI) program [8]. There was $100 \%$ homology with C. mucifaciens NML 97-0160 (GenBank accession no. AF537601), followed by two other sequences of C. mucifaciens (GenBank accession no. AF537600 and Y11200, respectively) with $99.1 \%$ and $98.0 \%$ similarity, respectively.

Strain NML 97-0160 is a blood isolate; it was described in GenBank as "closest to $C$. mucifaciens", with colonies that are smooth, not mucoid or yellow, and urease positive [2]. Colony description is consistent with the characteristics of our isolate; however, our strain was unable to hydrolyze urea.

\section{Discussion}

Identification of Corynebacterium to the species level is problematic. Recently, several species were renamed and some were even moved to other genera. Correct identification of these organisms would help us better understand the pathogenic potential of each Corynebacterium spp. and of related species.

Identification can be attempted by API Coryne; however, the database provided by the manufacturer is not complete, and some strains may be misidentified due to overlapping numerical codes. Additional numerical codes for recentlydescribed species are often found in published papers [4] or in microbiology manuals [1], which should be consulted

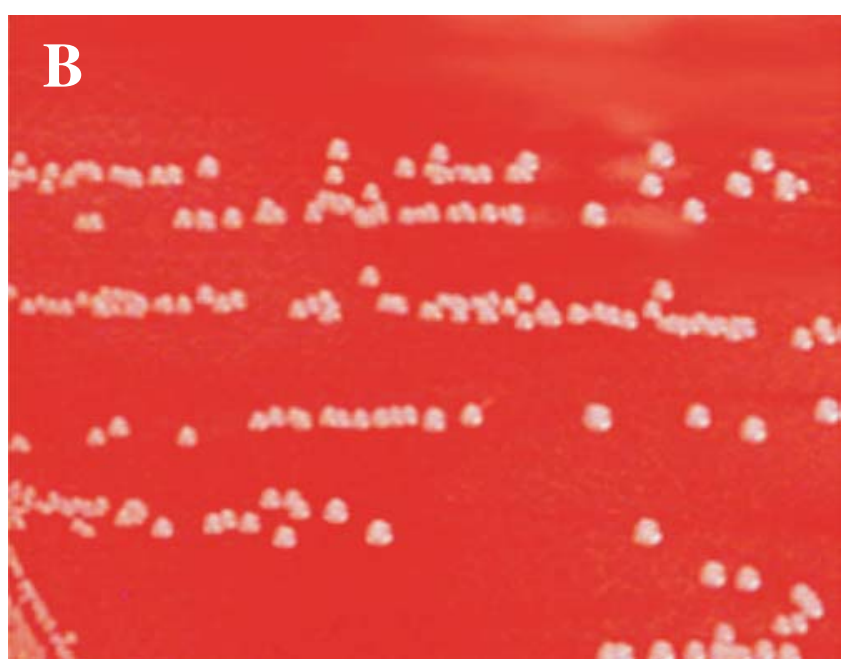

whenever an unusual Corynebacterium strain is isolated from a clinical sample. We suggest that results obtained using API Coryne should be carefully checked and compared with other microbiological information, such as colony morphology, color, and reference texts. Discrepancies must be checked, and other tests should be performed to assure the correct identification of the species. Preferably, DNA amplification and sequencing of target genes, such as $16 \mathrm{~S}$ rRNA, should be done to confirm discrepant results obtained with biochemical and commercial identification tests.

The increasing number of different species of Corynebacterium being reported also has implications for the treatment of infections due to these organisms. Weiss et al. [3] suggest that disk diffusion tests, following interpretation using streptococcus criteria, would be useful for assessing the activity of penicillin against Corynebacterium spp.

Corynebacterium mucifaciens is often isolated from blood or other normally-sterile body fluids $[1,4]$. In our case, we isolated it from a severely-ill patient who had several complicating factors. The patient died before results from blood cultures were available to guide antibiotic therapy.

In summary, we describe the isolation of a $C$. mucifaciens strain from blood cultures, which was correctly identified by API Coryne when the numerical code was checked in the reference literature $[1,4]$. However, this particular strain showed colony morphology and a partial $16 \mathrm{~S}$ rRNA sequence that were different from what is reported for reference strains [2]. The best match for the partial 16S rRNA sequence was strain NML 97-0160, described as "closest to C. mucifaciens"; but unlike our strain, it was urea positive. Since aberrant strains are likely to exist and may complicate the interpretation of 
phenotypic tests, molecular methods, such as analysis of $16 \mathrm{~S}$ rRNA gene sequences can provide a viable alternative for bacterial identification, especially for bacterial strains isolated from relevant clinical samples.

\section{References}

1. Funke G., Bernard K.A. Coryneform Gram-positive rods. In: Murray P.R., Barron E.J., Pfaller M.A., et al. eds. Manual of clinical microbiology, $7^{\text {th }}$ ed. American Society for Microbiology, Washington, D.C.: 1999.

2. Funke G., Lawson P.A., Collins M.D. Corynebacterium mucifaciens sp. nov., an unusual species from human clinical material. Int J Syst Bacteriol 1997;47:952-7.

3. Weiss K., Laverdière M., Rivest R. Comparison of antimicrobial susceptibilities of Corynebacterium species by broth microdilution and disk diffusion methods. Antimicrob Agents Chemother 1996;40:930-3.
4. Bernard K.A., Munro C., Wiebe D., Ongsansoy E. Characteristic of rare or recently described Corynebacterium species recovered from human clinical material in Canada. J Clin Microbiol 2002;40:4375-81.

5. Johnson G., Nelson S., Petric M., Tellier R. Comprehensive PCR-based assay for detection and species identification of human herpes viruses. J Clin Microbiol 2000;38:3274-9.

6. Kirschner P., Meier A. Böttger E.C. Genotypic identification and detection of mycobacteria-facing novel and uncultured pathogens. In: Persing D.H., Smith T.F., Tenover F.C., White T.J. eds. Diagnostic molecular microbiology principles and applications. American Society for Microbiology, Washington, D.C.: 1993.

7. Germer J.J., Rys P.N., Thornvilson J.N., Persing D.H. Determination of hepatitis $\mathrm{C}$ virus genotype by direct sequence analysis of products generated with the Amplicor HCV test. J Clin Microbiol 1999;37:2625-30.

8. Devulder G., Perriere G., Baty F., Flandrois J.P. BIBI, a bioinformatics bacterial identification tool. J Clin Microbiol 2003;4:1785-7. 